\title{
Análisis de las publicaciones biomédicas chilenas indizadas en PubMed, en los años 2008 y 2009
}

\author{
GLORIA VALDÉS S. ${ }^{1}$, FERNANDA PÉREZ G. ${ }^{1, a}$, HUMBERTO REYES B. ${ }^{2}$
}

\section{An analysis of Chilean biomedical publications in PubMed in the years 2008-2009}

Background: During the years 2008 and 2009, 1,191 biomedical articles authored by Chilean investigators working in Chile were indexed in PubMed. Aims: To evaluate the potential visibility of those articles, according to scientometric indexes of the journals where they were published. Methods: Those journals where the articles had been published were identified and each journal's Impact Factor (JIF), 5-year JIF, SCImago Journal Rank (SJR), SCImago Quartiles (Q) for 2010 and the Source Normalized Impact per Paper (SNIP) for 2008-2009 were identified. Results: Three hundred and twelve articles $(26,2 \%)$ were dedicated to experimental studies in animals, tissues or cells and they were classified as "Biomedicine", while 879 (73,8\%) were classified as "Clinical Medicine"; in both areas the main type of articles were original reports (90\% and $73.6 \%$, respectively). Revista Médica de Chile and Revista Chilena de Infectología concentrated the greater number of publications. Articles classified in Biomedicine were published more frequently in English and in journals with higher scientometric indexes than those classified in Clinical Medicine. Conclusions: Biomedical articles dealing with clinical topics, particularly case reports, were published mostly in national journals or in foreign journals with low scientometric indexes. It can be partly attributable to the authors' interest in reaching local readers. The evaluation of research productivity should combine several scientometric indexes, selected according to the field of research, the institution's and investigators' interests, with a qualitative and multifactorial assessment.

(Rev Med Chile 2015; 143: 979-986)

Key words: Bibliometrics; Biomedical research; Biomedical publications; Chile; Journals, medical.

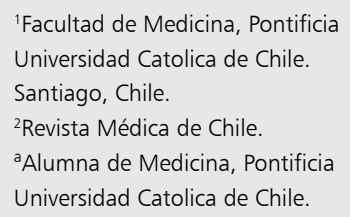

${ }^{1}$ Facultad de Medicina, Pontificia Universidad Catolica de Chile. Santiago, Chile.

${ }^{2}$ Revista Médica de Chile.

${ }^{a}$ Alumna de Medicina, Pontificia Universidad Catolica de Chile.

Conflictos de intereses: G. Valdés y F. Pérez no tienen nada que declarar. H. Reyes es Editor de la Revista Médica de Chile y fue excluido de la revisión de este manuscrito

Recibido el 7 de abril de 2015, aceptado el 9 de julio de 2015.

Correspondencia a: Dra. Gloria Valdés S. gvaldes@med.puc.cl
L a investigación científica es una actividad primordial de las universidades. La visibilidad de su producto final -las publicaciones científicas- es importante para los investigadores, para las instituciones académicas en que trabajan y las organizaciones que financian la investigación. Dado que es complejo valorar individualmente cada manuscrito, se ha generalizado la aplicación de índices cienciométricos que miden las citaciones recibidas por las revistas en que se publicaron, asumiendo que la visibilidad de las revistas puede aplicarse a todos los manuscritos publicados en ellas. El índice empleado con mayor frecuencia es el "factor de impacto", conocido con su sigla en inglés: JIF (Journal Impact Factor) y obtenido según el número de citas recibidas en un año determinado, dividido por el número de artículos publicados en la revista en los dos años precedentes. Este índice es generado anualmente por el Journal of Citation Reports del Institute of 
Scientific Information (ISI), actualmente Thomson Reuters. El SCImago Research Group, grupo de investigación que emplea datos de Scopus, calcula -entre otros- el SCImago Journal Rank (SJR). Las diferencias entre estos índices derivan de las distintas bases de datos empleadas y la metodología para calcularlos ${ }^{1}$. Además, hay que considerar el indicador "Source Normalized Impact per Paper" $(S N I P)$, también basado en Scopus, que corrige diferencias en las citaciones según disciplinas y áreas científicas específicas².

Un estudio previo analizó la investigación biomédica en Chile en el período 2007-2009, calificando como "investigación biomédica" a la investigación científica destinada a obtener nuevos conocimientos y proponer soluciones a problemas de salud de las personas y de la población ${ }^{3}$. En la base de datos MEDLINE/PubMed de los años 2008 y 2009 se identificaron 1.191 publicaciones ("artículos") generadas en Chile, realizadas por autores chilenos cuya pertenencia a entidades nacionales fue identificada y permitió cuantificar el origen institucional de las publicaciones.

El propósito del presente trabajo fue evaluar la calidad y visibilidad potenciales de esos artículos y sus diferencias según su tipología, empleando como variables sustitutas a los índices cienciométricos utilizados más frecuentemente por las universidades para calificar la producción científica de sus académicos, y por las entidades que financian la investigación.

\section{Material y Método}

\section{Muestra estudiada}

Se revisaron los 1.191 artículos obtenidos de MEDLINE/PubMed en los años 2008 y 2009, generados por autores chilenos adscritos a una institución nacional. Analizando sus títulos y los resúmenes en inglés, cada artículo fue clasificado en el área de: 1) Biomedicina, si incluyó estudios en animales o en tejidos, células o fracciones celulares, destinados al mejor conocimiento de la fisiología humana o relacionados con temas de la salud, y revisiones de temas con objetivos similares (en la publicación precedente $e^{3}$ esta área se denominó "investigación biomédica básica”); o de 2) Medicina Clínica, si incluyó estudios en seres humanos, epidemiológicos, seguimientos clínicos, ensayos clínicos controlados y no controlados, análisis retrospectivos, casos clínicos, revisiones de temas referidos a salud, salud pública, administración en salud y estudios de ciencias sociales orientados a salud ${ }^{3,4}$. Se identificaron las revistas en que se publicaron los artículos, las entidades que las editan, y el idioma de cada publicación.

\section{Definición de la tipología de los artículos}

Considerando los datos aportados en el resumen y la clasificación asignada en PubMed, los artículos se definieron como "artículos de investigación”, "revisiones de tema”, o "reportes de casos clínicos”. Se excluyeron del análisis las cartas al editor.

\section{Indicadores cienciométricos}

Para cada revista se aplicaron índices cienciométricos registrados en el año 2010, dando así la oportunidad de haber sido citados a los artículos publicados en el bienio 2008-2009. Del Journal of Citation Reports se obtuvo el JIF y el factor acumulado en 5 años (5-year JIF). De SCImago se obtuvieron el SCImago Journal Rank (SJR) y el cuartil (Q) correspondiente a la revista; en el caso de revistas con áreas temáticas con diferentes cuartiles se asignó el cuartil correspondiente al tema del artículo. El SNIP se obtuvo del Center for Science and Technology Studies Journal Indicator de la Universidad de Leiden ${ }^{5}$.

\section{Estadística}

Para comparar variables continuas en ambas áreas de la investigación biomédica se utilizó la prueba Mann-Whitney Rank Sum Test; las variables discontinuas se analizaron con test de $\chi^{2}$. El análisis se realizó mediante el programa GraphPad. Se consideraron significativas las diferencias con valor de $\mathrm{p}<0,05$.

\section{Resultados}

\section{Identificación de las revistas}

En PubMed se identificaron las 513 revistas que publicaron 1.191 artículos originados en Chile. En el área de Biomedicina, 312 artículos $(26,2 \%)$ se publicaron en 188 revistas ( 1 a 9 artículos por revista). En el área de Medicina Clínica, 879 artículos $(73,8 \%)$ se publicaron en 368 revistas ( 1 a 257 artículos por revista).

\section{Tipología de los artículos}

En Biomedicina, 281 (90\%) publicaciones correspondieron a "artículos de investigación" y 31 


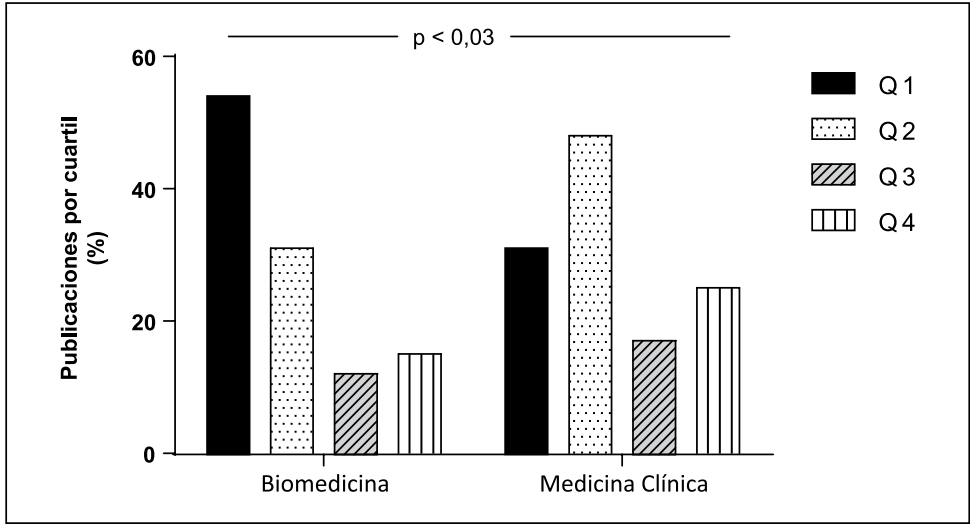

Figura 1. Proporción de publicaciones según cuartiles para las áreas de Biomedicina y Medicina Clínica.
(10\%) fueron "artículos de revisión". En Medicina Clínica, 647 (73,6\%) correspondieron a "artículos de investigación", 75 (8,5\%) a "artículos de revisión", 149 (16,9\%) a "reportes de casos" y 8 (1\%) fueron clasificados en PubMed como "artículos de revisión y reportes de casos". Los reportes de casos se publicaron mayoritariamente en Revista Médica de Chile (71), Revista Chilena de Infectología (18) y Transplantation Proceedings (6).

\section{Distribución de los artículos según los cuartiles de ubicación de las revistas}

La Figura 1 muestra la distribución de los artículos, por áreas, en los cuartiles (Q, SCImago) de las revistas que los publicaron. La proporción de artículos en revistas de los cuartiles superiores (Q1 y Q2) fue mayor en Biomedicina que en Medicina Clínica $(\mathrm{p}<0,03)$.

Índices cienciométricos de las revistas que publicaron mayor número de artículos

Veintisiete revistas publicaron 5 o más artículos chilenos cada una, reuniendo 524 artículos (43,9\% del total de artículos en el bienio). La Tabla 1 identifica estas 27 revistas, ordenadas según el número de artículos chilenos que publicaron, y muestra 6 de sus índices cienciométricos. Encabezan la nómina 3 revistas chilenas que concentraron $28,9 \%$ del total de los artículos.

La correspondencia entre los índices JIF y SNIP se evaluó mediante un test de correlación en las 27 revistas de la Tabla 1 mostrando una correlación elevada y significativa, pero variable entre los años estudiados $(\mathrm{R}=0,673 \mathrm{p}<0,001, \mathrm{n}$ $=23$ y $0,799 \mathrm{p}<0,001, \mathrm{n}=24)$ para los años 2008 y 2009 , respectivamente.

\section{Visibilidad a corto y mediano plazo}

Para el año 2010 el promedio del JIF anual y el acumulado en 5 años fue significativamente mayor en el área de Biomedicina que en Medicina clínica $(3,70 \pm 0,17$ y $3,82 \pm 0,18$ versus $1,6 \pm 0,08$ y $1,79 \pm 0,08 ; p<0,0001)$. La Figura 2 muestra el índice acumulado al año 2010. En ambas áreas hubo revistas de reciente incorporación al Journal of Citation Reports, por lo que no pueden exhibir visibilidad a 5 años.

En la Tabla 2 se identifican las 10 revistas con mayor JIF que publicaron artículos chilenos en el bienio 2008-2009.

\section{Idioma de las publicaciones}

La Tabla 3 muestra la distribución de los artículos según el idioma en que se publicó su texto completo. Tanto en las áreas de Biomedicina y

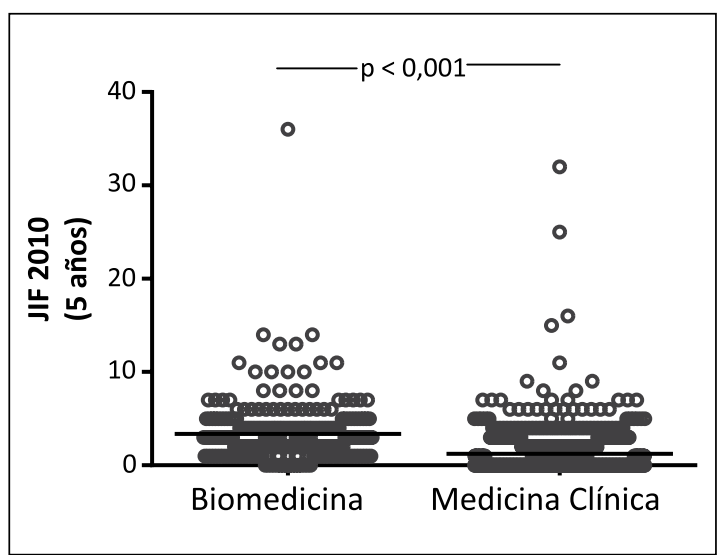

Figura 2. El factor de impacto 2010 acumulado en los 5 años previos para cada publicación está representado por un punto. La línea continua representa el promedio en cada área. 
Tabla 1. Revistas con más publicaciones biomédicas chilenas de 2008-2009

\begin{tabular}{|c|c|c|c|c|c|c|c|c|}
\hline Revista & $\mathbf{n}$ & $\begin{array}{c}\text { JIF } \\
2010\end{array}$ & $\begin{array}{l}\text { 5-year } \\
\text { JIF }\end{array}$ & $\begin{array}{c}\text { SJR } \\
08\end{array}$ & $\begin{array}{c}\text { SJR } \\
09\end{array}$ & $\begin{array}{l}\text { Q Sci- } \\
\text { mago }\end{array}$ & $\begin{array}{l}\text { SNIP } \\
2008\end{array}$ & $\begin{array}{l}\text { SNIP } \\
2009\end{array}$ \\
\hline Revista Médica de Chile & 257 & 0,37 & 0,47 & 0,20 & 0,20 & 2 & 0,31 & 0,48 \\
\hline Revista Chilena de Infectología & 63 & 0,36 & 0,00 & 0,16 & 0,15 & 3 & 0,26 & 0,32 \\
\hline Biological Research & 17 & 0,67 & 1,20 & 0,54 & 0,51 & 3 & 0,45 & 0,60 \\
\hline Obesity Surgery & 15 & 3,08 & 3,09 & 1,32 & 1,23 & 1 & 1,08 & 0,98 \\
\hline Transplantion Proceedings & 14 & 0,99 & 1,05 & 0,51 & 0,49 & 2 & 0,52 & 0,56 \\
\hline Archivos Españoles de Urología & 11 & 0,00 & 0,00 & 1,15 & 0,14 & 3 & 0,21 & 0,24 \\
\hline Archivos Latinoamericanos de Nutrición & 11 & 0,43 & 0,58 & 0,16 & 0,20 & 2 & 0,15 & 0,37 \\
\hline Actas Urológicas Españolas & 10 & 0,27 & 0,00 & 0,17 & 0,20 & 3 & 0,37 & 0,39 \\
\hline Current Vascular Pharmacology & 10 & 3,18 & 3,01 & 1,00 & 1,00 & 1 & 0,93 & 1,01 \\
\hline Fertility and Sterility & 9 & 3,12 & 3,48 & 1,17 & 1,13 & 1 & 1,19 & 1,2 \\
\hline Journal of Cellular Physiology & 9 & 3,99 & 4,22 & 2,28 & 2,47 & 2 & 1,08 & 1,17 \\
\hline Plastic and Reconstructive Surgery & 9 & 2,64 & 2,91 & 1,60 & 1,60 & 1 & 2,05 & 1,95 \\
\hline Gastroenterology and Hepatology & 7 & 0,00 & 0,00 & 1,18 & 0,13 & 3 & 0,06 & 0,05 \\
\hline Revista Panamericana de Salud Pública & 7 & 0,00 & 0,00 & 0,56 & 0,55 & 3 & 0,70 & 0,67 \\
\hline Allergologia et Immunopathologia & 6 & 0,78 & 0,00 & 0,27 & 0,27 & 3 & 0,54 & 0,38 \\
\hline Hormone Research in Pediatrics & 6 & 2,04 & 2,08 & 0,85 & 0,88 & 1 & & \\
\hline $\begin{array}{l}\text { Revista Española de Anestesiología y } \\
\text { Reanimación }\end{array}$ & 6 & 0,00 & 0,00 & 0,00 & 0,00 & 3 & & \\
\hline $\begin{array}{l}\text { Biochemical and Biophysical Research } \\
\text { Communications }\end{array}$ & 5 & 2,60 & 2,72 & 1,50 & 1,40 & 2 & 0,81 & 0,76 \\
\hline Bioorganic and Medicinal Chemistry & 5 & 2,98 & 3,11 & 1,19 & 1,11 & 1 & 1,25 & 1,34 \\
\hline Cardiovascular Research & 5 & 6,05 & 6,18 & 2,87 & 2,90 & 1 & 1,61 & 1,71 \\
\hline Clinical Chimica Acta & 5 & 2,39 & 2,64 & 1,03 & 0,90 & 2 & 1,25 & 1,17 \\
\hline Journal of Biological Chemistry & 5 & 2,60 & 2,72 & 1,49 & 1,40 & 2 & 1,37 & 1,34 \\
\hline Liver International & 5 & 3,84 & 3,34 & 1,10 & 1,10 & 1 & 0,99 & 1,12 \\
\hline Molecular Medicine Reports & 5 & 0,31 & 0,31 & 0,00 & 0,14 & 3 & & 0,16 \\
\hline Placenta & 5 & 2,99 & 3,06 & 1,19 & 1,12 & 1 & 1,02 & 1,01 \\
\hline Prenatal Diagnosis & 5 & 2,15 & 1,91 & 0,69 & 0,70 & 2 & 0,96 & 1,03 \\
\hline Reviews of Neurology & 5 & 0,53 & 0,56 & 1,22 & 1,30 & 3 & & \\
\hline
\end{tabular}

Medicina Clínica predominaron las publicaciones en inglés, lo que fue más marcado para los artículos de Biomedicina. Hubo escasas publicaciones en francés, portugués, italiano y alemán.

Los artículos de Biomedicina publicados en inglés se concentraron en revistas cuyo factor de impacto acumulado en 5 años fue mayor que el de las revistas que publicaron artículos de Medicina Clínica en inglés $(3,85 \pm 0,20$ versus $2,58 \pm 0,11$; $\mathrm{p}<0,0001)$.

\section{Origen geográfico de las revistas}

Los artículos fueron publicados por 184 casas editoriales en los Estados Unidos de Norteamérica; 
Tabla 2. Indicadores bibliométricos de las 10 revistas que publicaron el mayor número de artículos originados en Chile en 2008-2009

\begin{tabular}{lcccccc} 
Revista & n & JIF 2010 & 5-year JIF & SJR 08 & SJR 09 & Q Scimago \\
Área Biomedicina & & & & & & \\
Biological Research & 9 & 0,67 & 1,204 & 0,54 & 0,51 & 3 \\
Journal of Cellular Physiology & 8 & 3,986 & 4,217 & 2,28 & 2,47 & 2 \\
Biochemical and Biophysical Research Communications & 5 & 2,595 & 2,72 & 1,49 & 1,4 & 2 \\
Journal of Biological Chemistry & 5 & 5,328 & 5,498 & 4,3 & 4,1 & 1 \\
Antioxidants and Redox Signaling & 4 & 8,209 & 7,139 & 3 & 3 & 4 \\
Endocrinology & 4 & 4,993 & 5,265 & 2,51 & 2,38 & 1 \\
American Journal Physiology and Cell Physiology & 3 & 3,817 & 4,068 & 2,58 & 2,36 & 2 \\
Applied Biochemistry and Biotechnology & 3 & 1,879 & 2,053 & 0,55 & 0,66 & 3 \\
Biochimica Biophysica Acta & 3 & 0 & 0 & 2,28 & 2,28 & 2 \\
Biology of Blood and Marrow Transplantion & 3 & 3,275 & 3,587 & 1,21 & 1,12 & 1 \\
Área Medicina Clínica & & & & & & \\
Revista Médica de Chile & 256 & 0,366 & 0,47 & 0,2 & 0,2 & 2 \\
Revista Chilena de Infectología & 63 & 0,364 & & 0,16 & 0,15 & 3 \\
Obesity Surgery & 15 & 3,078 & 3,087 & 1,32 & 1,23 & 1 \\
Transplantion Proceedings & 14 & 0,993 & 1,046 & 0,51 & 0,49 & 2 \\
Archivos Españoles de Urología & 11 & 0 & 0 & 1,15 & 0,14 & 3 \\
Archivos Latinoamericanos de Nutrición & 11 & 0,427 & 0,582 & 0,16 & 0,2 & 2 \\
Actas Urológicas Españolas & 10 & 0,274 & & 0,17 & 0,2 & 3 \\
Biological Research & 9 & 0,67 & 1,204 & 0,54 & 0,51 \\
Plastic and Reconstructive Surgery & 9 & 2,635 & 2,911 & 1,16 & 1,16 \\
Current Vascular Pharmacology & 8 & 3,184 & 3,01 & 1 & 1 & 3 \\
\hline
\end{tabular}

http://www.scimagojr.com/journalsearch.php - https://es.scribd.com/doc/REV 78123026-Impact-Factor-Journal- Citation-Reports-by-Thomson-Reuters.

Tabla 3. Idioma en que fueron publicados los trabajos biomédicos según área de investigación

\begin{tabular}{|lcccc|}
\hline & $\mathbf{n}$ & $\begin{array}{c}\text { Inglés } \\
\mathbf{n ~ ( \% )}\end{array}$ & $\begin{array}{c}\text { Español } \\
\mathbf{n}(\%)\end{array}$ & $\begin{array}{c}\text { Otros } \\
\mathbf{n}(\%)\end{array}$ \\
\hline Biomedicina & 312 & $307(98,5)$ & $4(1,3)$ & $1(0,3)$ \\
Medicina clínica & 879 & $517(58,8)$ & $351(39,9)$ & $11(1,2)$ \\
\hline Total & 1.191 & $824(69,2)$ & $355(29,8)$ & $12(1,0)$ \\
\hline
\end{tabular}

$\chi^{2} 134.1852 ; p<0,00001$.

149 en Inglaterra; 50 en los Países Bajos; 24 en Alemania; 12 en Irlanda, Suiza y España; 11 en Brasil; 7 en Italia; 5 en Japón; 4 en Dinamarca, México y Francia; 3 en Chile y Grecia; 2 en China y Polonia; 1 en Argentina, Austria, Bangladesh, Colombia, Comunidad Europea, Federación Rusa, Finlandia o Venezuela.

\section{Discusión}

Este análisis de las revistas en que se publicaron artículos chilenos en el bienio 2008-2009 complementa al estudio previo sobre la investigación biomédica en Chile, que identificó a las instituciones en que se desarrollaron proyectos de investigación, sus fuentes de financiamiento (nacional y extranjeras) y los montos globales respectivos, las publicaciones generadas en ese período (identificadas en PubMed) y su origen institucional, y otras variables ${ }^{3}$. El presente estudio se centra en estimar la calidad y la visibilidad potenciales de esas publicaciones, aplicándoles los índices cienciométricos más frecuentemente utilizados en los análisis cuantitativos de la producción científica. Sus principales hallazgos son: 
1) Los 1.191 artículos chilenos se distribuyeron en 531 revistas, reflejando la diversidad de las ramas de las ciencias y de las especialidades clínicas; 2) Si bien hubo menos artículos en el área de Biomedicina que en Medicina Clínica, su mayor proporción se publicó en revistas extranjeras con mayor visibilidad (cuartiles 1 y 2 ) y permanencia de las citaciones, según las bases de datos Journal of Citation Reports, SCImago Journal Rank y Center for Science and Technology Studies (Leiden).

Un estudio reciente de SCImago señala que Medicina (según su categorización) es la segunda área científica en volumen de artículos generados en Chile y tiene una mayor cantidad de categorías temáticas que otras áreas ${ }^{6}$. Ese estudio muestra que en el período 2003 a 2012, entre 6.562 revistas donde publicaron investigadores chilenos de todas las áreas de las ciencias, el primer lugar en número de publicaciones lo ocupó Revista Médica de Chile, seguida por Revista Chilena de Pediatría $\left(6^{\circ}\right), R e-$ vista Chilena de Infectología $\left(8^{\circ}\right)$, Revista Chilena de Cirugía $\left(11^{\circ}\right)$, Revista Chilena de Obstetricia y Ginecología $\left(14^{\circ}\right)$ y Revista Chilena de Radiología $\left(25^{\circ}\right)$. Estos datos, unidos a los nuestros, destacan la importancia que tienen las revistas médicas chilenas para la difusión de artículos generados por investigadores nacionales, particularmente en Medicina Clínica.

El idioma de publicación en ciencias es importante por cuanto se ha comprobado que, cualquiera sea la naturaleza de un artículo científico, las posibilidades de recibir citaciones son mayores si se publica en inglés ${ }^{7-9}$. El reciente estudio de SCImago sobre la actividad científica chilena pone énfasis en que las citas recibidas por los artículos chilenos publicados en inglés, en revistas internacionales, es considerablemente mayor que el de los artículos publicados en español, sea en revistas editadas en Chile, España, México, Colombia o Argentina ${ }^{6}$. Un estudio en 7 prestigiosas revistas internacionales publicadas en inglés verificó que los artículos provenientes de Argentina, Brasil, Chile y México tenían Factores de Impacto significativamente menores que los procedentes del Reino Unido, Francia, Alemania, Japón y Estados Unidos de Norteamérica, lo que pudiera deberse a una menor calidad y relevancia o a un sesgo psico-social negativo ${ }^{7}$.

Creemos importante imaginar las causas que pudieron guiar a los autores chilenos al elegir publicar en revistas extranjeras o nacionales.
Una podría ser que los autores esperan interesar a distintos públicos lectores. En Biomedicina, los autores desean llegar a un público internacional, por la eventual universalidad de las materias que investigaron. Además, Biological Research, la principal revista chilena en esta área, se publica en inglés. En cambio, en Medicina Clínica los autores tendrían mayor interés por acceder a un público local o regional, por el contenido del artículo y su eventual repercusión en salud humana. Debemos considerar que, si bien estas revistas chilenas reciben menos citaciones, son revistas de "corriente principal", con accesibilidad universal. Otras causas posibles incluyen la facilidad para redactar manuscritos en el idioma materno y la mayor benevolencia de la revisión editorial en revistas chilenas que en las revistas ubicadas en el cuartil superior: la Revista Médica de Chile y la Revista Chilena de Infectología tienen alrededor de $30 \%$ de rechazos, comparadas con más de $70 \%$ en las revistas del cuartil 1.

Debemos recalcar que hubo artículos de $\mathrm{Me}$ dicina Clínica que se publicaron en español y en revistas extranjeras cuyos índices cienciométricos no difieren sustancialmente de las revistas nacionales. Algunos tratan materias de subespecialidades médicas que no cuentan con revistas chilenas incluidas en bases de datos internacionales.

Nuestro trabajo tiene limitaciones:

$1^{\mathrm{a}}$ : Los datos se obtuvieron exclusivamente de PubMed. Si bien es la base de datos más utilizada en las revisiones de la literatura médica y científica, esta selección dejó fuera del análisis a publicaciones que están indizadas en otras bases de datos importantes (Ejs: SciELO y LILACS). Entre las revistas nacionales quedó excluida la Revista Chilena de Pediatría, incorporada a PubMed con posterioridad al bienio 2008-2009 y que en el estudio de SCImago ocupa el segundo lugar entre las revistas chilenas por el número de artículos publicados anualmente ${ }^{6}$.

$2^{\mathrm{a}}$ : Los índices cienciométricos utilizados corresponden al conjunto de artículos publicados en cada revista, pero no identifican el número de citaciones que recibieron individualmente sus artículos. Las instituciones que emplean la producción en publicaciones como uno de los parámetros principales para calificar académicos, financiar proyectos de investigación o acreditar programas universitarios, deberían considerar que al emplear 
los "rankings" de las revistas científicas están traspasando a los autores los atributos de las revistas donde publicaron, sin dejar de considerar que los autores han logrado satisfacer los estrictos criterios de selección de las revistas con alto "ranking".

Se han diseñado alternativas para corregir esta situación. El "índice H”, propuesto por Hirsch en el año $2005^{10}$, considera las citaciones que reciben individualmente los autores de artículos científicos, lo que tendría mayor precisión para evaluar el impacto individual de un investigador y sería útil para predecir sus expectativas de éxito en una carrera académica ${ }^{11}$. Aunque ha sido validado para publicaciones relacionadas al área de salud, adolecería de limitaciones, entre las cuales está el número de años dedicados a la investigación, favoreciendo a los investigadores más antiguos ${ }^{12}$.

Debemos destacar que en este estudio hemos analizado índices cienciométricos que forman parte fundamental de los antecedentes requeridos por las entidades que financian proyectos de investigación, y por las universidades cuando califican a sus académicos. Como consecuencia de ello la ubicación de las revistas en "rankings" establecidos mediante estos índices - particularmente el JIF- es utilizado por muchos autores para elegir dónde enviar sus artículos a publicación.

3: Haber analizado datos obtenidos en un período corto y alejado: 2008-2009. El objetivo central de este trabajo fue complementar las observaciones de una publicación previa, en que se estudiaron otras características de la investigación biomédica en Chile ${ }^{3}$. Las publicaciones analizadas en este trabajo representan $71 \%$ de las publicaciones citables de Medicina Clínica del año 2013 contabilizadas por el SCImago Country Journal Rank, lo cual nos permite suponer que las conclusiones derivadas de la muestra identificada en 2008-2009 pueden extrapolarse a una muestra tomada en el 2013, el último año registrado en la base SCImago.

En ámbitos de las ciencias y de las humanidades se ha criticado reiteradamente el uso de los índices cienciométricos para evaluar la relevancia de las publicaciones y calificar el desempeño académico de sus autores ${ }^{13}$. Esta situación condujo en el año 2012 a una Declaración Pública, respaldada por 78 organizaciones científicas y más de un centenar de académicos de un gran número de países ${ }^{14}$. Recientemente, el "Manifiesto de Leiden para Métricas de la Investigación” presenta un “decálogo" de principios para guiar la evaluación de la investigación, distinguiendo requisitos para las agencias que proveen financiamiento, para las instituciones que patrocinan la investigación y califican a los participantes, para las revistas que la publican, para los organismos que generan las métricas y para los investigadores ${ }^{15}$. Una de las principales enseñanzas de ambos documentos ${ }^{14,15}$ es que para evaluar la productividad en investigación de cada persona se deberían considerar varios elementos de juicio, cuanti y cualitativos, que incluyen su antigüedad en este proceso, el área específica de la temática a que se dedica, los índices cienciométricos pertinentes a ello y la relevancia potencial de cada manuscrito para el medio local o el mundo científico en general.

Recomendamos a los autores -particularmente en el área de Medicina Clínica- que cuando elijan la revista a la que enviarán un manuscrito, consideren sus índices cienciométricos, el universo de lectores a los que aspiran acceder y el impacto que podría tener su publicación en las decisiones clínicas y en el diseño de políticas de salud.

\section{Referencias}

1. Falagas ME, Kouranos VD, Arencibia JR, Karageorgopoulos DE. Comparison of SCImago journal rank indicator with journal impact factor. FASEB J 2008; 22: 2623-8.

2. Measuring contextual citation impact of scientific journals. [http://arxiv.org/abs/0911.2632]

3. Valdés SG, Armas MR, Reyes H. Principales características de la investigación biomédica en Chile. Rev Med Chile 2012; 140: 484-92.

4. Fletcher RH, Fletcher SW: Clinical research in general medical journals: a 30-year perspective. N Engl J Med 1979; 301: 180-3.

5. [http://www.journalindicators.com/methodology]

6. CONICYT, SCImago (Eds.): Principales indicadores cienciométricos de la actividad científica chilena 2012. Informe 2014: una mirada a 10 años. Madrid-Valparaíso: Altazor; 2014.

7. Estrada-Mejía C, Forero-Pinedo C. The quest for visibility of scientific journals in Latin America. Learned Publishing 2010; 23: 237-52.

8. Vinther S, Rosenberg J. Impact factor trends for general medical journals: non-English language journals are lagging behind. Swiss Medical Weekly 2012; 142: w13572. 
9. van Leeuwen ThN, Moed HF, Tijssen RJW, Visser MS, van Raan AFJ. Language biases in the coverage of the Science Citation Index and its consequences for international comparison of national research performance. Scientometrics 2001; 51: 335-46.

10. Hirsch JE. An index to quantify an individual's scientific research output. Proc Natl Acad Sci USA 2005; 102: 16569-72.

11. Hirsch JE. Does the $\mathrm{H}$ index have predictive power? Proc Natl Acad Sci USA 2007; 104: 19193-8.

12. Patel VM, Ashrafian H, Ahmed K, Arora S, Jiwan S,
Nicholson JK, et al. How has healthcare research performance been assessed?: A systematic review. J R Soc Med 2011; 104: 251-61.

13. Ossa J. El elitismo del “semáforo" académico. El Mercurio 12 de noviembre de 2014.

14. San Francisco Declaration on Research Assessment. [http://www.ascb.org/dora-old/files/SFDeclarationFINAL.pdf]

15. Hicks D, Wouters P, Waltman L, de Rijcke S, Rafols I. Bibliometrics: The Leiden Manifesto for research metrics. Nature 2015; 520: 429-31. 